\title{
DESIGN AND IN VITRO EVALUATION OF MULTIPARTICULATE SYSTEM FOR THE CHRONOMODULATED DELIVERY OF LORNOXICAM
}

\author{
Patil Shrishailgouda $S^{1,3 *}$, Gupta Venkatadri Ram Mohan ${ }^{2,3}$ \\ ${ }^{1}$ Department of Pharmaceutics, N.E.T Pharmacy College, Raichur, Karnataka, India \\ ${ }^{2}$ Department of Pharmaceutics, Pulla Reddy Institute of Pharmacy, Dommadugu, Dundigal, Hyderabad, Telangana, \\ India \\ 3Department of Pharmaceutical Sciences, Jawaharlal Nehru Technological University, Kukatpally, Hyderabad, \\ Telangana, India
}

Received 12 March 2015; Review Completed 07 April 2015; Accepted 02 May 2015, Available online 15 May 2015

\begin{abstract}
:
In view of chronobiological considerations of rheumatoid arthritis, the present study deals with the design and evaluation of multiparticulate system for the chronomodulated delivery of lornoxicam. The basic design is based on the Pulsincap technology and consisted of formaldehyde treated insoluble hard gelatin capsule body filled with tripolyphosphate cross-linked chitosan microspheres of lornoxicam and sealed with a hydrogel tablet plug. The entire device was enteric coated, so as to prevent the variable gastric emptying time. The microspheres were found to be discrete, nearly spherical with rough surface characteristics. Particle size, encapsulation efficiency and release rate of lornoxicam from the microspheres are dependent on the fabrication conditions of the microencapsulation. FT-IR, DSC and XRD studies revealed the compatibility of the drug with the excipients used. The exposure of hard gelatine capsule bodies with formaldehyde for a period of $12 \mathrm{hrs}$ was found to be optimum in maintaining the intactness of the capsule bodies in the simulated GI fluids. The lag time of the designed delivery system varied from 3-7 hrs and depended on the nature and concentration of the plugging material. The ejection of the plug was delayed with increase in the polymer concentration. Among the two polymers studied as plugging materials, guar gum exhibited better control in maintaining the lag time compared to xanthan gum. From the study it can be concluded that, the lag time be efficiently tailored by varying the concentrations of the polymers in the hydrogel plug for the chronomodulated delivery of lornoxicam.
\end{abstract}

Key Words: Rheumatoid arthritis; Lornoxicam; Microspheres; Chronomodulated delivery systems; Hydrogel plug; Lag time.

\section{INTRODUCTION:}

Due to advances in chronobiology, chronopharmacology and global market constraints, the traditional goal of pharmaceutics, that is designing drug delivery system with a constant drug release rate is becoming obsolete for certain diseases. Nowadays, concept of chronopharmaceutics has emerged, wherein, research is devoted to the design and evaluation of drug delivery systems that release a therapeutic agent at a rhythm that ideally match the biological requirement of a given disease therapy ${ }^{1}$. By timing drug administration, plasma peak is obtained at an optimal time and the number of doses per day can be reduced. Saturable first-pass metabolism and tolerance development can also be avoided $^{2}$. The diseases currently targeted for chronomodulated formulations include asthma, arthritis, duodenal ulcer, cancer, diabetes, cardiovascular diseases, hypercholesterolemia, ulcer and neurological diseases ${ }^{3}$.

Morning stiffness associated with pain at the time of awakening is a diagnostic criterion of the rheumatoid arthritis (RA). In patients with RA, inflammation induced changes in synovial fluid composition, oedema of the synovium and periarticular structures as well as redistribution of interstitial fluid while sleeping, contribute to clinical stiffness of the joints that is most prominent in the morning. Chronopharmacotherapy for RA has been recommended to ensure that the highest blood levels of the drug coincide with peak pain and stiffness ${ }^{4}$.

Lornoxicam, a congener of tenoxicam, is a novel NSAID belonging to the oxicam group ${ }^{5}$ with extremely potent anti-inflammatory and analgesic activities ${ }^{6,7}$. It is widely used for the symptomatic treatment of pain and inflammation in patients with rheumatoid arthritis and osteoarthritis $^{8}$.

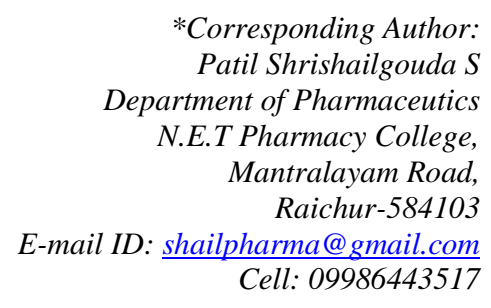

*Corresponding Author: tment of Pharmaceutics Mantralayam Road, raichur-584103 Cell: 09986443517 
However, lornoxicam's usefulness is limited due to its short half-life that ranges from 3 to $5 h^{9,10}$. Due to its rapid elimination rate, frequent administration of lornoxicam is needed to achieve long lasting and constant pain relief.

In our previous studies we have reported the $\mathrm{pH}$ dependent solubility of lornoxicam which indicated better solubility in the higher $\mathrm{pH}$ pertaining to the colonic region ${ }^{11}$. Hence, in light of these facts, it is desirable to design the delivery system that produces the peak drug levels that ideally that matches the cirrcadiam rhythum of the rheumatoid arthritis resulting in better therapeutic outcome.

Compared to single-unit dosage forms, multiparticlate systems like microspheres exhibit more uniform distribution and absorption of the drug in the gastrointestinal tract, reduced local irritation, higher colonic residence time, more predictable gastric emptying and also eliminates unwanted intestinal retention of polymeric material ${ }^{12,13}$. Chitosan is an interesting biopolymer to prepare microspheres owing to its unique polymeric cationic character, good biocompatibility, non-toxicity, biodegradability, mucoadhesiveness and also due to its absorption enhancing effect ${ }^{14,15}$. Thus, the present study deals with the design and evaluation of multiparticulate system for the chronomodulated delivery of lornoxicam with an aim to achieve desired levels of the drug in the early morning hours for the effective management of RA. The chronomodulated delivery system was based on modified Pulsincap technology using TPP cross-linked chitosan microspheres loaded with lornoxicam and suitable hydrogel plugs.

\section{MATERIALS AND METHODS:}

Chitosan (Chitoclear®, 95\% deacetylated) was obtained as gift sample from Primex Ltd, Iceland. Lornoxicam pure drug was generously supplied by Sun Pharma Ltd., Vadodhara. TPP was purchased from Molychem Ltd Hyderabed. Guar gum and xanthan gum were purchased from Himedia Laboratories Pvt Ltd, Mumbai. Hard gelatine capsules were generously supplied by Elegant Drugs Pvt Ltd, Hubli, Karnataka. All other chemicals and reagents were of analytical grade and were purchased from SD fine chemicals, Mumbai.

\section{Preparation of microspheres:}

Lornoxicam loaded chitosan microspheres were prepared by ionic gelation technique using TPP as crosslinking agent. Briefly, $2 \% \mathrm{w} / \mathrm{v}$ chitosan solution was prepared using $1 \%$ aqueous acetic acid by stirring overnight on a magnetic stirrer. Accurately weighed quantity of lornoxicam was dispersed uniformly into the chitosan solution using the magnetic stirrer for $2 \mathrm{~h}$ and further subjected for ultasonication $(15 \mathrm{~min})$ to remove the air bubbles. The drug containing chitosan solution was dropped into the gently agitated aqueous TPP solution with a disposable syringe $(22 \mathrm{G})$ at the rate of $1 \mathrm{ml} / \mathrm{min}$ using digital over head stirrer. Microspheres were instantaneously formed and after predetermined period of time, microspheres were decanted from the dispersion medium and washed several times with distilled water to remove any surface adhering TPP. The microspheres thus obtained were dried at room temperature for $24 \mathrm{~h}$ and stored in a desiccator for further use $^{16,17}$. The details of formulation and process variables studied are given Table No: 1

\section{Characterization of Microspheres}

\section{Particle size and surface topography}

The size of the prepared microspheres was analysed using optical microscopy fitted with a calibrated eye piece micrometer. The mean of 100 microspheres was noted as average particle size ${ }^{18}$. The surface topography of the microspheres was studied using scanning electron microscopy (JEOL, JSM-6360, Japan). Microspheres were mounted on aluminium specimen stubs using double sided adhesive tape and coated with platinum under vacuum. The morphology of the microspheres was observed at acceleration voltage of $10 \mathrm{kV}$ at different magnifications.

\section{Fourier tranform -infrared (FT-IR) spectral studies:}

Pure drug, drug-excipients physical mixture and drug loaded microspheres were analyzed using Fourier transformer infrared spectrophotometer (IR Affinity 1 model, Shimazdu Corporation, Japan, with diffuse reflectance scan sampling method). The samples were triturated with $\mathrm{KBr}$ and scanned over wave number range of 4000 to $400 \mathrm{~cm}^{-1}$. FT-IR spectra were analyzed for functional groups and drug polymer interactions.

\section{$\mathrm{X}$-ray diffraction (XRD) analysis:}

The effect of microencapsulation process on drug crystallinity was studied using XRD analysis. XRD patterns of pure drug, physical mixture and microspheres were recorded on X-ray diffractometer (XRD 6000, Shimadzu corporation, Japan) using Nifiltered, CuK radiation, a voltage of $40 \mathrm{Kv}$ and current of $30 \mathrm{~mA}$. The scanning speed employed was $4 \% \mathrm{~min}$ over the $10^{\circ}$ to $80^{\circ}$ diffraction angle range. Microspheres were triturated to fine powder before performing the analysis.

\section{Differential scanning calorimetric (DSC) studies:}

The pure drug, drug-excipients physical mixture and drug loaded microspheres were subjected to differential scanning calorimeter (DSC, SIECKO, model-6300, Japan). The samples were sealed in aluminium pans and heated at a constant rate of $10^{\circ} \mathrm{C} / \mathrm{min}$ over a temperature range of $20-300{ }^{\circ} \mathrm{C}$. Inert atmosphere was maintained by purging nitrogen gas at a flow rate of $10 \mathrm{ml} / \mathrm{min}$.

Encapsulation efficiency: Crushed microspheres equivalent to $8 \mathrm{mg}$ of lornoxicam was accurately weighed and transferred to a $100 \mathrm{ml}$ volumetric flask containing $50 \mathrm{ml}$ of phosphate buffer of $\mathrm{pH} 7.4$ and the volume was made up to the mark using the same buffer solution. The flask was stirred on a thermostatic water bath (Remi Equipments Ltd., Mumbai) at room temperature for $24 \mathrm{~h}$ to extract the entrapped drug. The content was filtered and after suitable dilution, the absorbance was noted on a UV spectrophotometer (UV1700, Shimadzu Corporation, Japan) at $377.0 \mathrm{~nm}$ using phosphate buffer of $\mathrm{pH} 7.4$ as blank. Triplicate readings for each batch were noted and the average was determined as drug content of the microspheres ${ }^{16,18}$. 


\section{In vitro drug release:}

The release of lornoxicam from the microspheres was studied using USP type II dissolution apparatus (TDT08L, Electrolab dissolution tester). Microspheres equivalent to $8 \mathrm{mg}$ of lornoxicam were taken into the basket and the release studies were carried out under the following conditions; media: $900 \mathrm{ml}$ of phosphate buffer of pH 6.8; temperature: $37 \pm 0.5{ }^{\circ} \mathrm{C}$; speed: $100 \mathrm{rpm}$. At fixed interval of time, aliquots were withdrawn and replaced with fresh dissolution media to maintain the constant volume. The concentration of drug released at different time intervals was then determined by measuring the absorbance at $376.5 \mathrm{~nm}$ against blank using UV spectrophotometer.

Table 1: Composition, Yield, Encapsulation Efficiency and Particle size of Lornoxicam loaded chitosan microspheres $(\mathbf{n}=3)$

\begin{tabular}{|c|c|c|c|c|c|c|}
\hline & Variables & & Yield (\%) & $\begin{array}{l}\text { Actual Drug } \\
\text { content }(\mathrm{mg})^{*}\end{array}$ & $\begin{array}{l}\text { Encapsulation } \\
\text { Efficiency }(\%)\end{array}$ & $\begin{array}{l}\text { Particle Size } \\
(\mu \mathrm{m})\end{array}$ \\
\hline Code & Core: coat & \multirow{5}{*}{$\begin{array}{l}\text { constant: } \\
2 \% \text { TPP, } \\
\text { pH } 8.5, \\
30 \text { min }\end{array}$} & & & & \\
\hline $\mathrm{C} 1$ & $1: 2$ & & $78.57(2.11)$ & $4.51(0.14)$ & $56.33(1.8)$ & $762.66(7.41)$ \\
\hline $\mathrm{C} 2$ & $1: 4$ & & $80.3(1.96)$ & $4.84(0.21)$ & $60.45(2.65)$ & $794.99(6.47)$ \\
\hline $\mathrm{C} 3$ & $1: 6$ & & $83.21(1.46)$ & $5.22(0.14)$ & $65.29(1.76)$ & $829.35(5.12)$ \\
\hline $\mathrm{C} 4$ & $1: 8$ & & $85.48(1.59)$ & $5.75(0.14)$ & $71.86(1.84)$ & $855.14(2.83)$ \\
\hline & $\begin{array}{l}\mathrm{pH} \text { of the } \\
\text { medium }\end{array}$ & \multirow{4}{*}{$\begin{array}{l}\text { constant: } \\
1: 8, \\
2 \% \mathrm{TPP}, \\
30 \mathrm{~min}\end{array}$} & & & & \\
\hline $\mathrm{H} 1$ & 6.0 & & $86.39(1.36)$ & $6.03(0.18)$ & $75.32(2.27)$ & 789.73(7.9) \\
\hline $\mathrm{H} 2$ & 4.0 & & $87.25(1.43)$ & $6.45(0.25)$ & $80.59(3.13)$ & $741.24(7.91)$ \\
\hline H3 & 2.0 & & $89.58(1.28)$ & $7.08(0.16)$ & $88.46(2.05)$ & $733.05(6.01)$ \\
\hline & TPP \% (w/v ) & \multirow{4}{*}{$\begin{array}{l}\text { constant: } \\
1: 8, \\
\text { pH } 2.0, \\
30 \text { min }\end{array}$} & & & & \\
\hline $\mathrm{P} 1$ & 4 & & $92.28(1.56)$ & $7.3(0.11)$ & $91.29(1.47)$ & $719.22(8.82)$ \\
\hline $\mathrm{P} 2$ & 6 & & $94.89(1.69)$ & $7.68(0.10)$ & $95.97(1.29)$ & $702.81(9.6)$ \\
\hline $\mathrm{P} 3$ & 8 & & 97.44(1.48) & $7.7(0.13)$ & $96.3(1.67)$ & $678.32(5.55)$ \\
\hline & $\begin{array}{l}\text { Reaction time } \\
(\mathrm{min})\end{array}$ & \multirow{4}{*}{$\begin{array}{l}\text { constant: } \\
1: 8, \\
8 \% \text { TPP, } \\
\text { pH } 2.0\end{array}$} & & & & \\
\hline $\mathrm{T} 1$ & 45 & & $97.58(1.55)$ & $7.73(0.11)$ & $96.59(1.43)$ & $659.25(4.63)$ \\
\hline $\mathrm{T} 2$ & 60 & & $97.19(1.7)$ & $7.63(0.11)$ & $95.39(1.45)$ & $621.67(7.19)$ \\
\hline $\mathrm{T} 3$ & 90 & & $96.45(1.41)$ & $7.29(0.29)$ & $91.19(3.62)$ & $615.32(3.21)$ \\
\hline
\end{tabular}

\section{Design of the chronotherapeutic system ${ }^{19}$ :}

The design of the chrnotherapeutic system involved following steps:

i. Cross-linking of hard gelatine capsules: About 100 hard gelatin capsules of size ' 2 ' were taken and their bodies and caps were separated. Then, the bodies were treated with formaldehyde vapours to render them insoluble in gastro intestinal fluids while the caps remained untreated. Twenty-five millilitres of $15 \%(\mathrm{v} / \mathrm{v})$ formaldehyde was taken into desiccator and a pinch of potassium permanganate was added to it to generate formalin vapors. The empty bodies of hard gelatin capsule were placed over wire mesh and then exposed to formaldehyde vapors. The desiccator was tightly closed thereby exposing the capsule bodies to formaldehyde vapours for the specified period of time (4-12hrs) and dried at $50^{\circ} \mathrm{C}$ for $30 \mathrm{~min}$ to ensure complete reaction between gelatin and formaldehyde vapors. The bodies were then dried at room temperature for another $12 \mathrm{hrs}$ to facilitate removal of residual formaldehyde. The formaldehyde treated bodies were joined with untreated caps and stored in a polythene bag.

ii. Optimization of formaldehyde treatment: The disintegration of the capsule bodies depend on the duration of formaldehyde exposure. Hence, crosslinking time was optimized by exposing the capsules bodies to the formaldehyde vapours for 4,8 and 12 hrs. The bodies and the untreated caps were joined and subjected for disintegration test (Electrolab ED2L, Servewell Instruments Pvt Ltd, Bengaluru). The untreated gelatine capsules were also subjected for dinintegration study for the comparison purpose. The disintegration test was carried out for 2, 3 and up to $24 \mathrm{hrs}$ in $0.1 \mathrm{~N} \mathrm{HCl}$, phosphate buffer of $\mathrm{pH} 6.8$ and 7.4 respectively. The time at which the capsules disintegrated were noted.

iii. Preparation of Hydrogel plug: Direct compression method was used to prepare the hydrogel tablet plug. The composition of different tablet plugs used in the design of chronotherapeutic systems is shown in Table 2. Natural polymers like guar gum and xanthan gum along with other excipients were utilized in the preparation of the plug. The plug ingredients (polymers and lactose) were mixed for 10 minutes using mortar and pestle. Magnesium stearate $(2 \% \mathrm{w} / \mathrm{w})$ was added to the previous mixture and further blended for 5 minutes and compressed using Rotary compression machine (Rimek Mini Press-1, Karnavathi Engineering Ltd, Gujarat).

iv. Capsule filling, plugging, sealing and enteric coating: Optimized Microspheres equivalent to $8 \mathrm{mg}$ of lornoxicam was manually loaded in to the bodies by hand filling. After filling with the microspheres, the mouth of the formaldehyde treated bodies were plugged with the prepared hydrogel plugs and closed with the untreated caps. The joint of the body and cap 
of the capsules was sealed with a small amount of $5 \%$ ethyl cellulose ethanolic solution. The sealed capsules were enteric coated by dip coating method with $4 \%$ HPMCP 55 in $4: 1 \quad(\mathrm{v} / \mathrm{v})$ mixture of dichloromethane:acetone, plasticized with dibutylphthalate $(0.75 \%)$, to prevent variable gastric emptying $^{20}$. Coating was repeated until an $8-12 \%$ increase in weight is obtained. \% weight gain of the capsules before and after coating was determined.

Table 2: Composition of tablet plugs using guar gum and xanthan gum as lag time modifiers.

\begin{tabular}{|c|c|c|c|c|c|}
\hline $\begin{array}{c}\text { Batch } \\
\text { code }\end{array}$ & GG & XG & $\begin{array}{c}\text { Spray Dried } \\
\text { Mannitol }\end{array}$ & $\begin{array}{c}\text { Mg. } \\
\text { Stearate }\end{array}$ & $\begin{array}{c}\text { Total } \\
\text { Weight }\end{array}$ \\
\hline LG1 & 60 & --- & 38 & 2 & 100 \\
\hline LG2 & 50 & --- & 48 & 2 & 100 \\
\hline LG3 & 40 & --- & 58 & 2 & 100 \\
\hline LG4 & 30 & & 68 & 2 & 100 \\
\hline LX1 & --- & 60 & 38 & 2 & 100 \\
\hline LX2 & --- & 50 & 48 & 2 & 100 \\
\hline LX3 & --- & 40 & 58 & 2 & 100 \\
\hline LX4 & --- & 30 & 68 & 2 & 100 \\
\hline
\end{tabular}

\section{Evaluation of Chronotherapeutic system:}

\section{Test for formaldehyde treated empty capsule bodies:}

Various physical tests such as, identification attributes, visual defects, dimension changes, solubility studies were carried out on the formaldehyde treated empty capsule bodies.

\section{Qualitative chemical test for free formaldehyde:}

Standard formaldehyde solution used is formaldehyde solution $(0.002 \mathrm{w} / \mathrm{v})$ and sample solution is prepared by dissolving formaldehyde treated bodies (about 25 in number) in distilled water. The content was stirred for 1 $\mathrm{h}$ with a magnetic stirrer, to solubilize the free formaldehyde. The solution was then filtered into a 50 $\mathrm{ml}$ volumetric flask, washed with distilled water and volume was made up to $50 \mathrm{ml}$ with the washings. In brief, to $1 \mathrm{ml}$ of sample solution, $9 \mathrm{ml}$ of distilled water was added. One milliliter of resulting solution was taken into a test tube and mixed with $4 \mathrm{ml}$ of water and $5 \mathrm{ml}$ of acetone reagent. The test tube was warmed in a water bath at $40{ }^{\circ} \mathrm{C}$ and allowed to stand for $40 \mathrm{~min}$. The solution was not more intensely colored than a reference solution prepared at the same time and in the same manner using $1 \mathrm{ml}$ of standard solution in place of the sample solution. The comparison was made by examining tubes down their vertical axis.

In vitro release studies: Dissolution studies were carried out by using USP XXIV dissolution apparatus (paddle method). Capsules were tied to paddle with a cotton thread so that the capsule should be immersed completely in dissolution media but not float. In order to simulate the $\mathrm{pH}$ changes along the GI tract, three dissolution media with $\mathrm{pH} 1.2,7.4$ and 6.8 were sequentially used, referred to as sequential $\mathrm{pH}$ change method. When performing experiments, the $\mathrm{pH} 1.2$ medium was first used for $2 \mathrm{~h}$ (since the average gastric emptying time is $2 \mathrm{~h}$ ), then removed and the fresh $\mathrm{pH}$
7.4 phosphate buffer saline (PBS) was added. After $3 \mathrm{~h}$ (average small intestinal transit time is $3 \mathrm{~h}$ ), the medium was removed and fresh phosphate buffer of $\mathrm{pH} 6.8$ was added for subsequent hours. Nine hundred milliliters of the dissolution medium was used at each time. Rotation speed was $100 \mathrm{rpm}$ and temperature was maintained at $37 \pm 0.5{ }^{\circ} \mathrm{C}$. Five milliliters of dissolution media was withdrawn at predetermined time intervals and fresh dissolution media was replaced. The withdrawn samples were analyzed at $372.5 \mathrm{~nm}, 376.5 \mathrm{~nm}$ and $377.5 \mathrm{~nm}$ for acidic medium, phosphate buffer of $\mathrm{pH} 6.8$ and 7.4 respectively by UV absorption spectroscopy and the cumulative percentage release was calculated over the sampling times.

\section{RESULTS AND DISCUSSION:}

Particle Size: The average particle size of the microspheres is given in Table No 1. Particle size increased with increase in core: coat ratio which could be due to more amount of coat material in same volume of liquid droplet. Whereas particle size decreased for the microspheres prepared at lower $\mathrm{pH}$ of TPP solution which could be attributed to the $\mathrm{pH}$ dependent ionization of TPP resulting in more ionically cross-linked microspheres. Particle size also reduced with an increase in the cross-linking agent concentration as well as extent of cross-linking ${ }^{21}$.

SEM photographs revealed that the prepared microspheres were discrete, broadly spherical with rough surface characteristics (Fig. 1). The surface of the microspheres was dense without any porous structures. The morphological studies also revealed the presence of few cracks on the surface of the microspheres. Our observations are concordant with the findings of other researchers who reported rough surface characteristics of chitosan microspheres prepared by the ionotropic gelation technique using TPP as cross-linking agent ${ }^{22,23}$. 


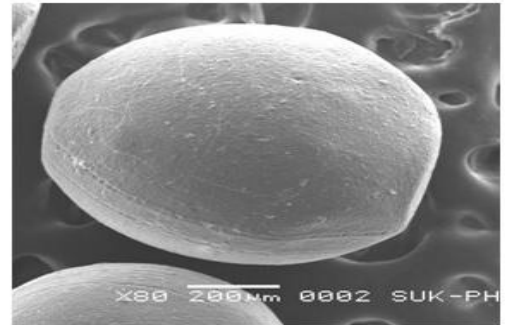

A

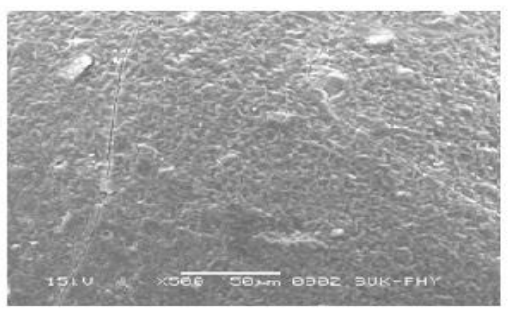

$\mathrm{C}$

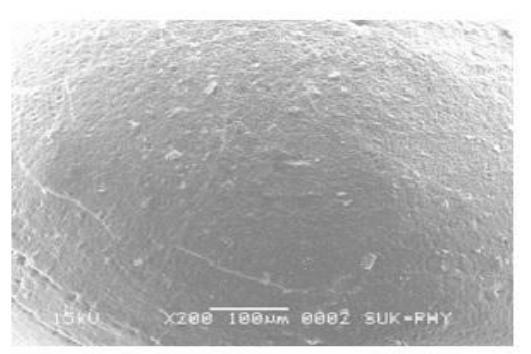

B

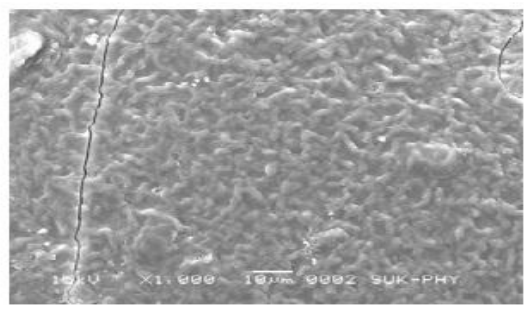

D

Figure 1: SEM Photographs of TPP cross-linked chitosan microspheres. A. Individual microsphere and its magnified surface topography B. 200X, C. 500X and D. 1000X.

\section{Encapsulation efficiency:}

The encapsulation efficiency increased with increase in core: coat ratio which could be due to more availability of active TPP binding sites in the polymeric chains leading to higher encapsulation efficiency. When the $\mathrm{pH}$ of the dispersion medium was changed from its original alkaline, a significant improvement in the lornoxicam entrapment was observed which may be due to its poor solubility in lower $\mathrm{pH}^{11}$. The encapsulation efficiency was also increased for the microspheres prepared at higher TPP concentrations. However, slight reduction in the entrapment of lornoxicam was noted when the curing time was increased from 30 to 90minutes.

\section{FT-IR studies:}

The FT-IR spectra of physical mixture, drug loaded microspheres were compared with the FT-IR spectrum of pure drug (Fig: 2). The pure drug lornoxicam exhibited its characteristic absorption bands at 3099.61 $\mathrm{cm}^{-1}$ due to aromatic/heterocyclic $\mathrm{C}-\mathrm{H}$ stretching and at $2926.01 \mathrm{~cm}^{-1}$ due to $\mathrm{C}-\mathrm{H}$ stretching of $\mathrm{CH}_{3}$ group. The other prominent absorption bands appeared at 1647.21 $\mathrm{cm}^{-1}$ due to stretching vibrations of $\mathrm{C}=\mathrm{O}$ of $\mathrm{CONH}$, 1618.28 due to aromatic $\mathrm{C}=\mathrm{C}$ and $\mathrm{C}=\mathrm{N}$ stretching. Bending vibrations of $\mathrm{NH}$ group in the secondary amine were observed at 1593.20 and $1544.96 \mathrm{~cm}^{-1}$ whereas aromatic $\mathrm{C}=\mathrm{C}$ skeleton stretching vibrations were observed at $1500.62 \mathrm{~cm}^{-1}$. The peaks at $1423.47 \mathrm{~cm}^{-1}$ and $1327.03 \mathrm{~cm}^{-1}$ corresponds to $\mathrm{O}=\mathrm{S}=\mathrm{O}$ group, $790 \mathrm{~cm}^{-1}$ due to substituted aromatic rings, $765.74 \mathrm{~cm}^{-1}$ due to $\mathrm{C}$ $\mathrm{Cl}$ stretching vibrations. The IR spectra of physical mixture and drug loaded microsphere formulation exhibited all the characteristic absorption bands as that of pure drug lornoxicam without any significant variations. It is clear from the spectra that, the drug has not undergone any kind of interactions with the excipients used and retained its identity both in its physical mixture as well as in its formulation.

\section{XRD studies:}

The XRD patterns of lornoxicam, its physical mixture and drug loaded microspheres are shown in Fig: 3 . The X-Ray powder diffractogram of pure drug exhibited a series of intense peaks which is indicative of its crystalline nature. The typical crystalline peaks of the drug in the physical mixture were clearly visible without any significant changes in the positions and relative intensities, thereby ruling out any interaction between the drug and the excipients. However, the diffractogram of the lornoxicam loaded microspheres showed peaks of diminished intensities indicating that the drug is molecularly dispersed in the polymeric matrix or might have undergone amorphization during the microsphere preparation $^{21}$.

DSC Studies: Differential Scanning Calorimetric analysis was also performed in order to establish the identity and integrity of drug in its pure form, physical mixture and also in the microsphere formulation. The thermogram of the pure drug lornoxicam showed an exothermic peak exhibiting its sharp melting point at $231.8{ }^{\circ} \mathrm{C}$ which corresponds to its reported range ${ }^{24}$. The thermogram of the physical mixture revealed the existence of the lornoxicam exothermic peak at $228.6{ }^{\circ} \mathrm{C}$ indicating the absence of interaction between the drug and the excipients. However, noticeable broadening of the peak intensity was observed which could be due to the presence of the formulation excipients. The thermogram of drug loaded microspheres revealed the disappearance of the characteristic exothermic peak of lornoxicam. This suggests that, the drug was molecularly dispersed in the polymeric matrix of the microspheres or existed in an amorphous form. This fact was also well supported by our XRD studies. From the study, it can be concluded that there is no interaction of the drug with the polymer and other excipients used in the formulation. 


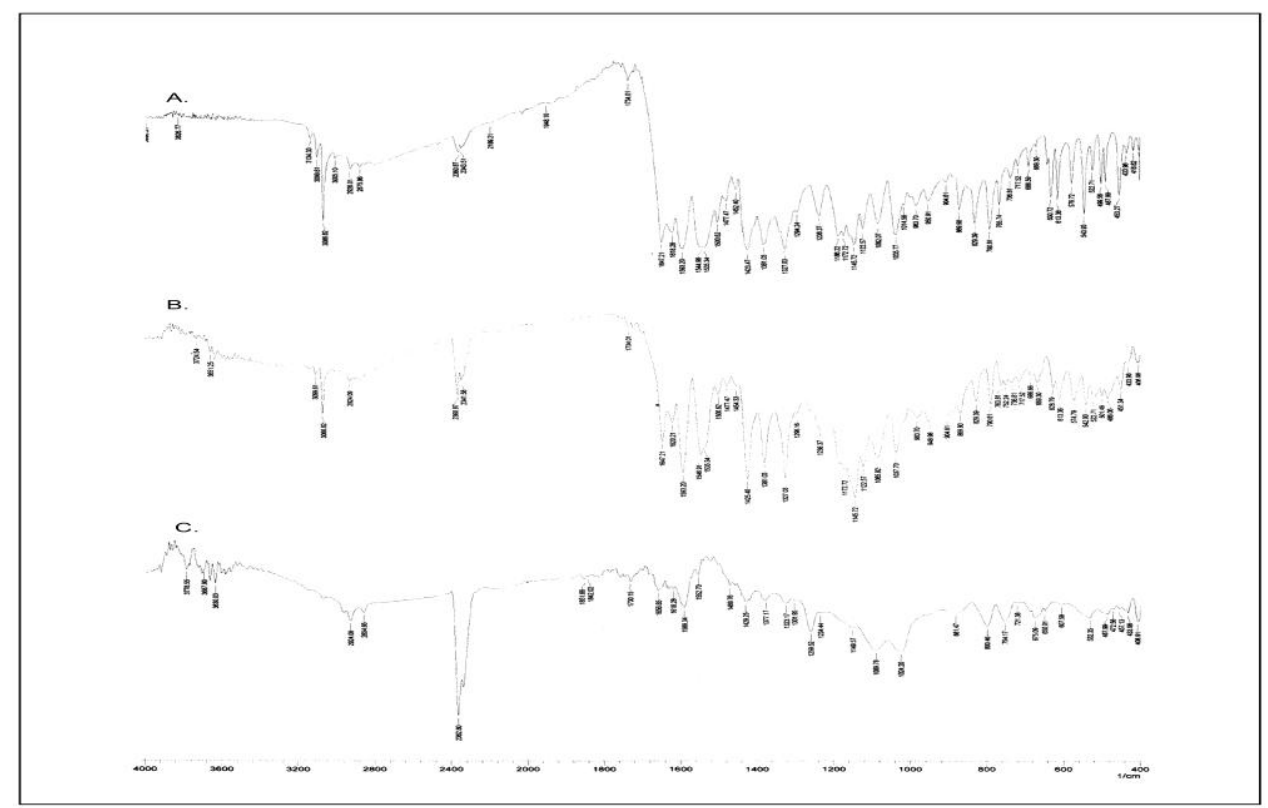

Figure 2: FT-IR Spectra of A. Pure drug lornoxicam, B. Physical Mixture, C. Drug loaded microspheres.

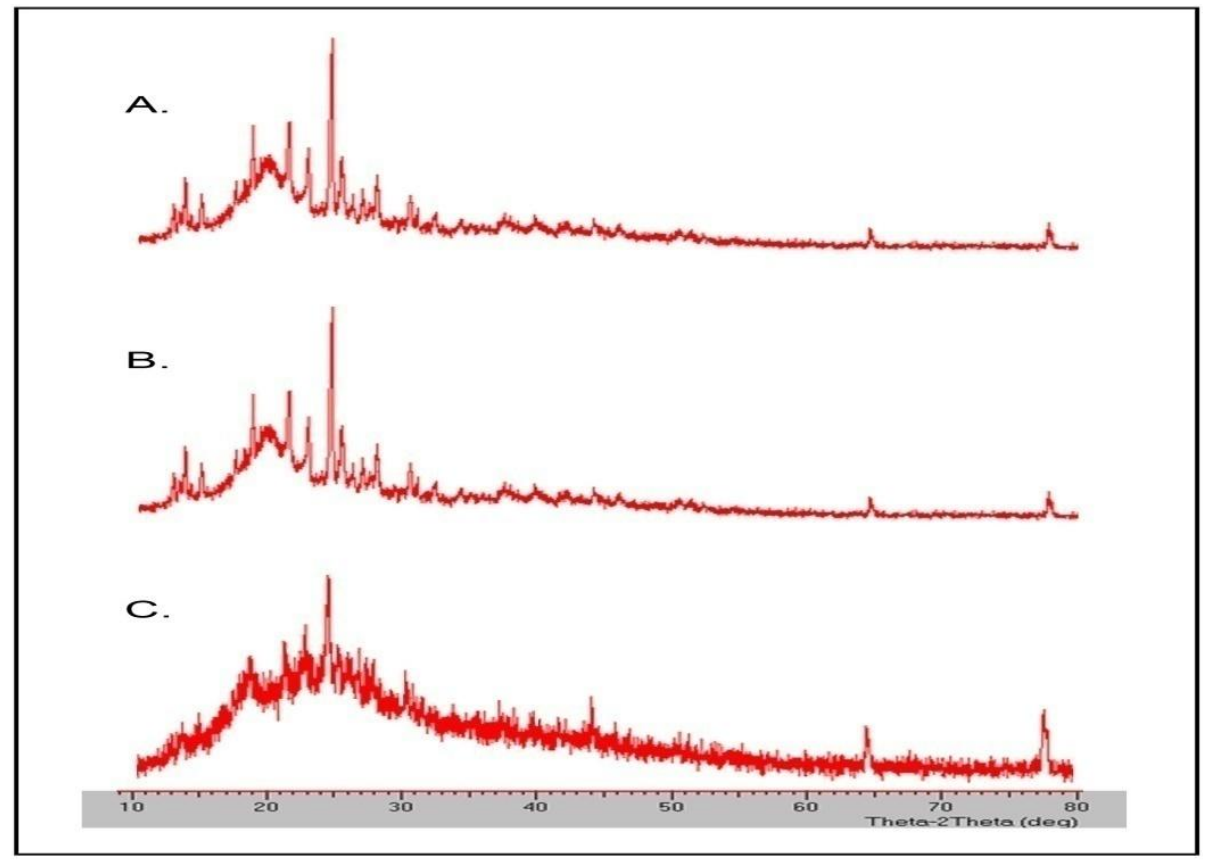

Figure 3: XRD Patterns of A. Pure drug lornoxicam, B. Physical Mixture, C. Drug loaded microspheres

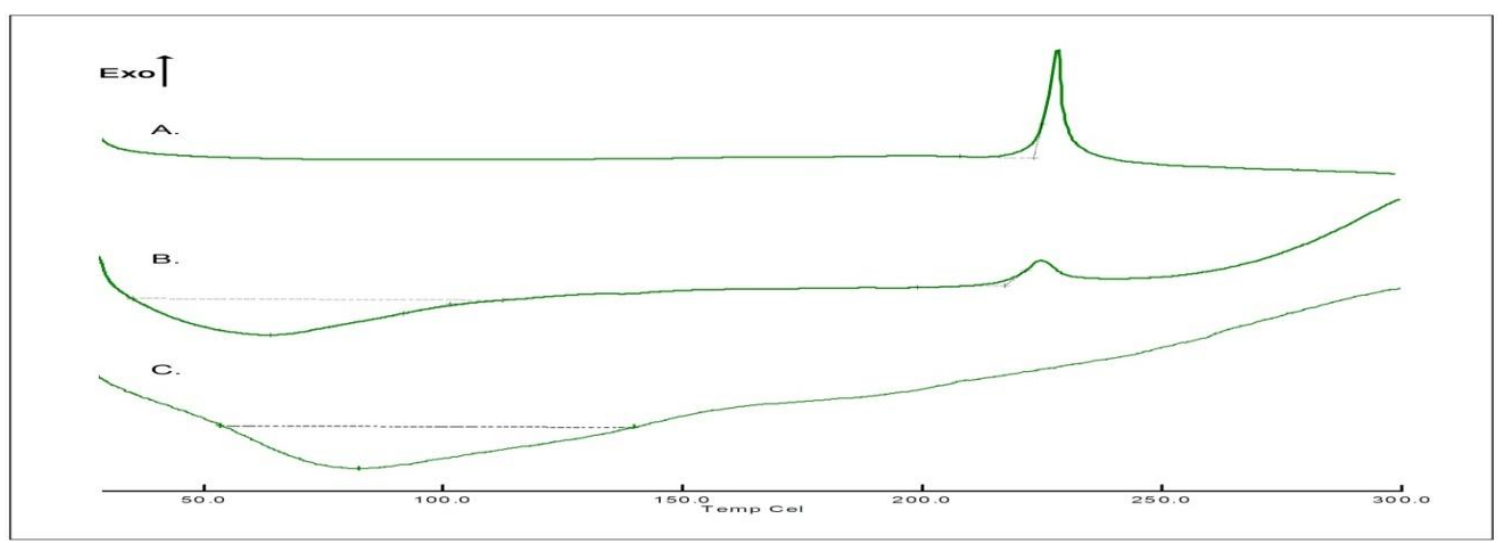

Figure 4: DSC Thermograms of A. Pure drug lornoxicam, B. Physical Mixture, C. Drug loaded microspheres. 


\section{In vitro drug release studies:}

Drug release from the TPP cross-linked chitosan microspheres was largely influenced by formulation (core: coat ratio and TPP concentration) and process ( $\mathrm{pH}$ of the dispersion medium and reaction time) variables. The plots of cumulative percentage drug release versus time for different formulations are shown in Fig No 5 and 6 . Initially, the microspheres prepared with various core: coat ratios and at fixed TPP concentration $(2 \% \mathrm{w} / \mathrm{v}), \mathrm{pH}$ of the dispersion medium (original $\mathrm{pH} 8.5$ ) and reaction time $(30 \mathrm{~min})$ were subjected for dissolution studies. Dissolution studies revealed the decrease in drug release with increasing core: coat ratio in the microspheres. This could be due to more efficient entanglement of the drug within the polymeric matrix of the microspheres composed of higher levels of coat material.

The results of the dissolution study of the microspheres prepared at different $\mathrm{pH}$ conditions revealed that, as the $\mathrm{pH}$ of the dispersion medium was lowered from its original $\mathrm{pH} 8.5$ to $\mathrm{pH} 2.0$, the drug release from the microspheres drastically reduced. On the other hand, microspheres prepared at higher $\mathrm{pH}$ exhibited faster drug release. This could be due to the fact that, at lower $\mathrm{pH}$, ionization degree of TPP and chitosan are high and complete cross-linking occurs without deprotonation ${ }^{22}$, thereby leading to more controlled drug release. The drug release from the microspheres proportionately decreased with increase in cross-linking agent concentration and duration of cross-linking. This could be due to more cross-linking of the polymeric chains with increased TPP concentration and also increased duration of microsphere exposure to the coagulant medium would provide more reaction time which leads to more efficient cross-linking and thus reducing the drug release. Overall, the results of dissolution studies revealed that, drug release from the microspheres can be efficiently tailored by altering the formulation and process parameters. Microspheres of $\mathrm{H} 2$ batch with release rate of more than $60 \%$ at the end of $6^{\text {th }} \mathrm{hr}$ and continued till the end of 12 hrs was selected for the design of chronomodulated delivery of lornoxicam. The selected batch also exhibited good encapsulation efficiency and surface characteristics.

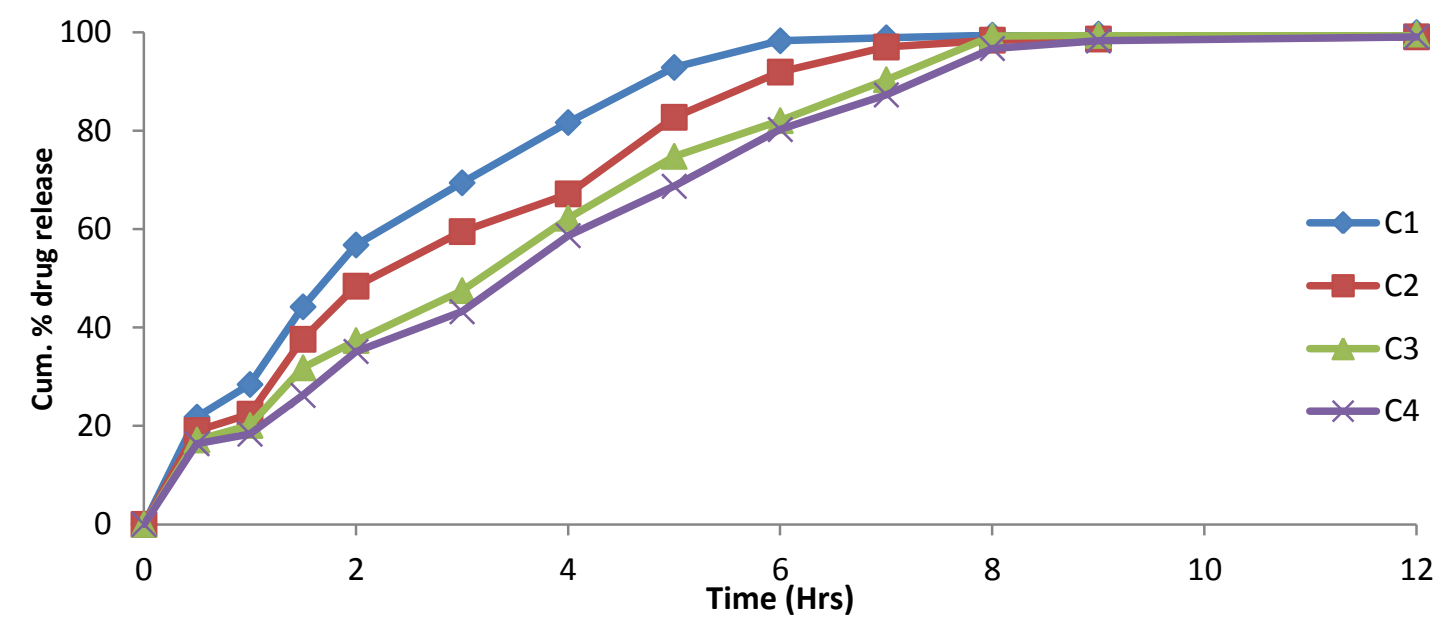

Figure 5: Effect of core: coat ratio on in vitro release of lornoxicam from TPP cross-linked chitosan microspheres.

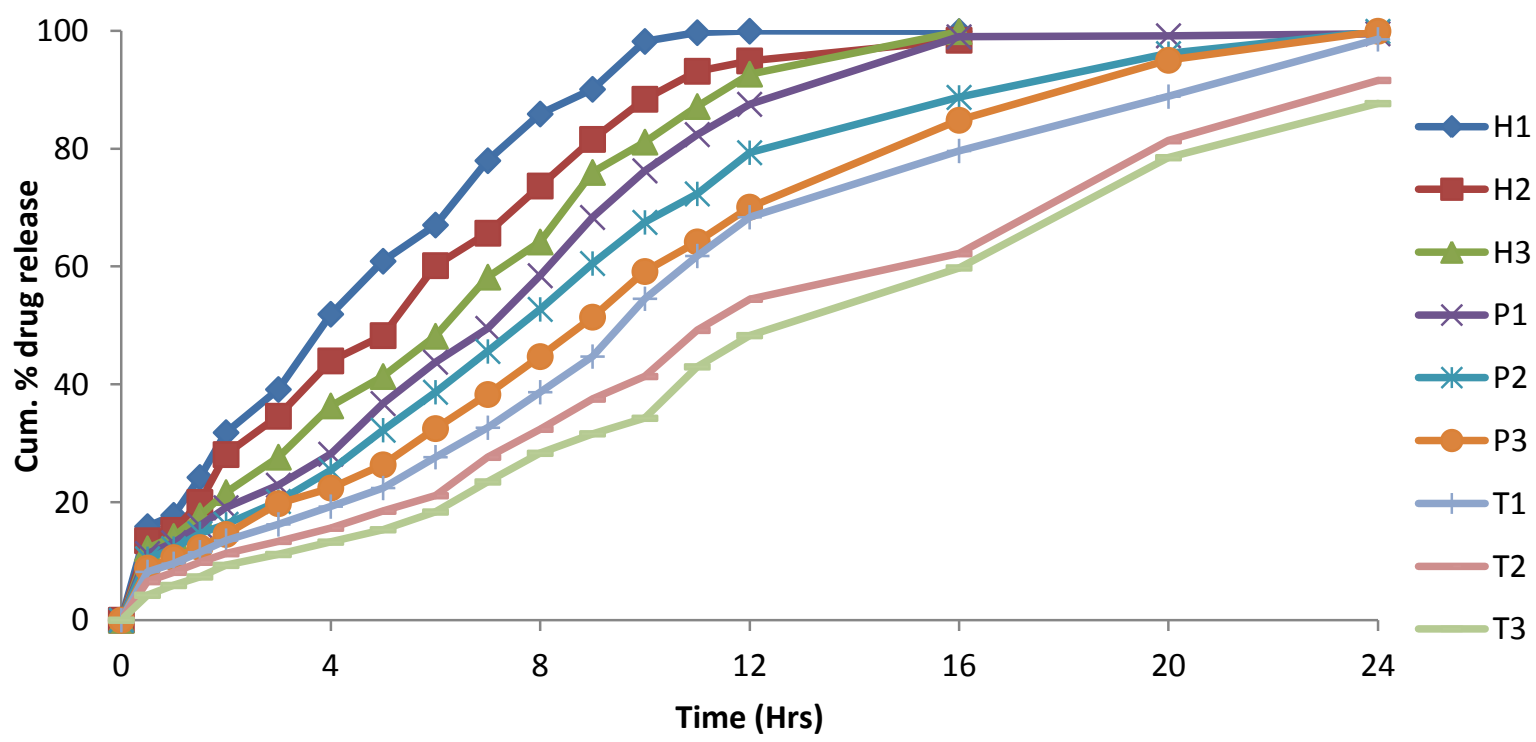

Figure 6: Effect of fabrication conditions on in vitro release of lornoxicam from TPP cross-linked chitosan microspheres. 
Mechanism of drug release: The drug release mechanism was studied with respect to zero order, first order, Higuchi model and Korsemayer and Peppas models. The coefficient values were found to be higher for Higuchi model (0.9952-0.9666) than Peppas model (0.9899-0.8903) followed by first order (0.9668-0.8517) and zero order (0.9907-0.8848) which is an indicative of diffusion controlled drug release. The $\mathrm{n}$ values of Peppas equation were in the range of 0.4031-0.8938 suggesting the anomalous (non-Fickian) release mechanism.

\section{Evaluation of chronomodulated system:}

\section{Formaldehyde treated empty capsule bodies:}

Formaldehyde treatment has been employed to modify the solubility of the gelatin capsules. Exposure to formalin vapors results in an unpredictable decrease in solubility of gelatin owing to the cross linkage of the amino groups in the gelatin molecular chain with aldehyde groups of formaldehyde by Schiffes base condensation. In about 100 capsule bodies treated with formaldehyde, about 8-10 bodies were found to be shrunk or distorted. Slight decrease in length and diameter of the capsule bodies was observed after treatment with the formaldehyde. The formaldehyde capsules were also tested for the presence of free formaldehyde. The sample solution was not more intensely colored than the standard solution inferring that less than $20 \mu \mathrm{g} / \mathrm{ml}$ of free formaldehyde per 25 capsules, taken for test.

\section{Optimization of cross-linking conditions:}

From the disintegration studies it was observed that, the untreated capsules (both body and cap) dissolved within 10-12 minutes where as the dissolution of the treated bodies depended upon the duration of cross-linking with formaldehyde. The capsule bodies treated for $4 \mathrm{hrs}$ with formaldehyde vapours become soft and sticky mass was formed at the end of $2 \mathrm{hrs}$ disintegration study in $0.1 \mathrm{~N}$ $\mathrm{HCl}$ solution and completely dissolved within $1 \mathrm{hr}$ when exposed to phosphate buffer of $\mathrm{pH}$ 6.8. The capsule bodies exposed to formaldehyde vapours for $8 \mathrm{hrs}$ did not disintegrated but become soft at the end of $10 \mathrm{hrs}$ disintegration study. However, the bodies treated with formaldehyde for $12 \mathrm{hrs}$ remained intact throughout the disintegration study of $24 \mathrm{hrs}$ and were selected for the design of chronotherapeutic systems. Various other workers have also adopted $12 \mathrm{hrs}$ formaldehyde treatment in the efficient cross-linking of the hard gelatine capsules $^{25}$.

In vitro release studies: All the designed chronotherapeutic systems of lornoxicam with guar gum and xanthan gum as plugging materials were subjected for the dissolution studies and the results are depicted in Fig 7and 8. From the graphs it is clear that, all the capsules remained intact in the acidic $\mathrm{pH}$ for the initial 2 hrs of the dissolution studies, indicating the integrity of the enteric coating with HPMCP. Further, when the

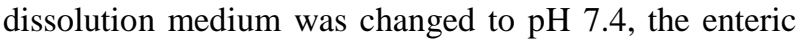
coating along with the soluble cap dissolved thereby exposing the hydrogel plug to the dissolution medium. The exposed hydrogel plug then, absorbed the surrounding fluid, swelled and released the drug through the swollen matrix. After this, the hydrogel plug turned out into a soft mass, which was then easily ejected out of the capsule body there by releasing the lornoxicam loaded chitosan microspheres in the simulated colonic fluid (phosphate buffer of $\mathrm{pH}$ 6.8). With all the formulations, there was absolutely no drug release in $\mathrm{pH}$ 1.2 , thus indicating the efficacy of enteric coating with HPMCP.

The polymer nature and concentration had profound impact on the swelling and ejection of the hydrogel plug. Irrespective of the polymer used, as its concentration was increased, there was a proportionate delay in the wetting and also the complete ejection of the plug.

Guar gum (GG) as a hydrogel plugging material in four different concentrations like 60, 50, 40 and $30 \mathrm{mg}$ were used in the formulations LG1, LG2, LG3 and LG4 respectively. The cumulative drug release at the end of $7^{\text {th }}, 6^{\text {th }}, 5^{\text {th }}$ and $4^{\text {th }}$ hr was found to be $4.56,3.35,6.18$ and 9.58\% with LG1, LG2, LG3 and LG4 respectively after which the plug were completely ejected and all the microspheres were released. It was observed that, as the polymer concentration in the hydrogel plug was increased, there was a proportionate delay in the ejection of the hydrogel plug. This could be attributed to delayed wetting and swelling of the hydrogel material at the higher polymeric concentration. Overall, with all the formulations containing guar gum as a plugging material, a lag period of 4-7 hrs was achieved and there after the drug release was spread over a period of $18 \mathrm{hrs}$ in a controlled manner.

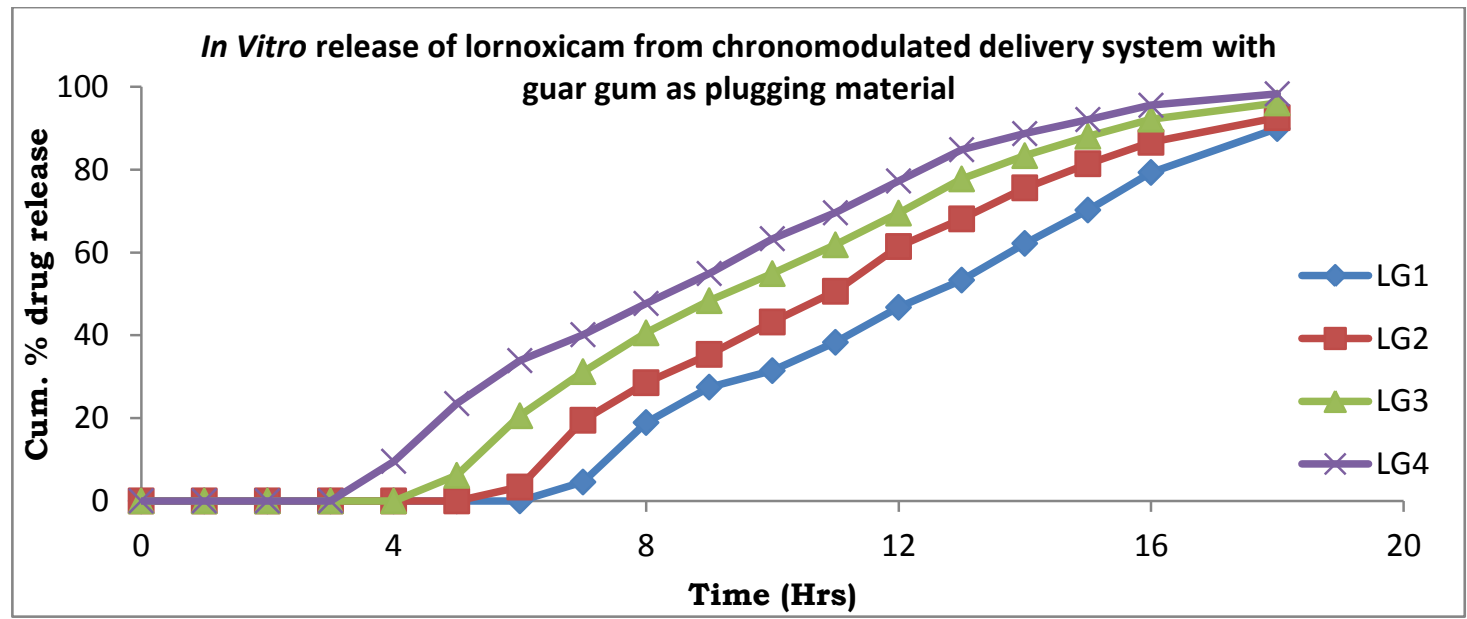


In another set of formulations, xanthan gum as a hydrogel plugging material in four different concentrations like 60, 50, 40 and $30 \mathrm{mg}$ were used in LX1, LX2, LX3 and LX4 respectively. The cumulative drug release at the end of $6^{\text {th }}$ and $5^{\text {th }} \mathrm{hr}$ was found to be 8.33 and $10.56 \%$ with LX1 and LX2 respectively after which the plug were completely ejected, whereas 7.33 and $5.88 \%$ drug was released at the end of $4^{\text {th }} \mathrm{hr}$ and $3^{\text {rd }}$ hr dissolution study in case of LX3 and LX4 respectively. Similarly as observed with guar gum, increase in xanthan gum concentration resulted in proportionate delay in the ejection of the hydrogel plug. However, the lag time observed in case of xanthan gum was shorter when compared to that of guar gum. This could be probably due to higher swelling nature of xanthan gum leading to early ejection of the plug when compared to the guar gum. Overall, from the study it can concluded, guar gum and xanthan gum as plugging materials provides the concentration depended lag time for the chronomodulated delivery of lornoxicam that could be utilized in the better management of rheumatoid arthritis.

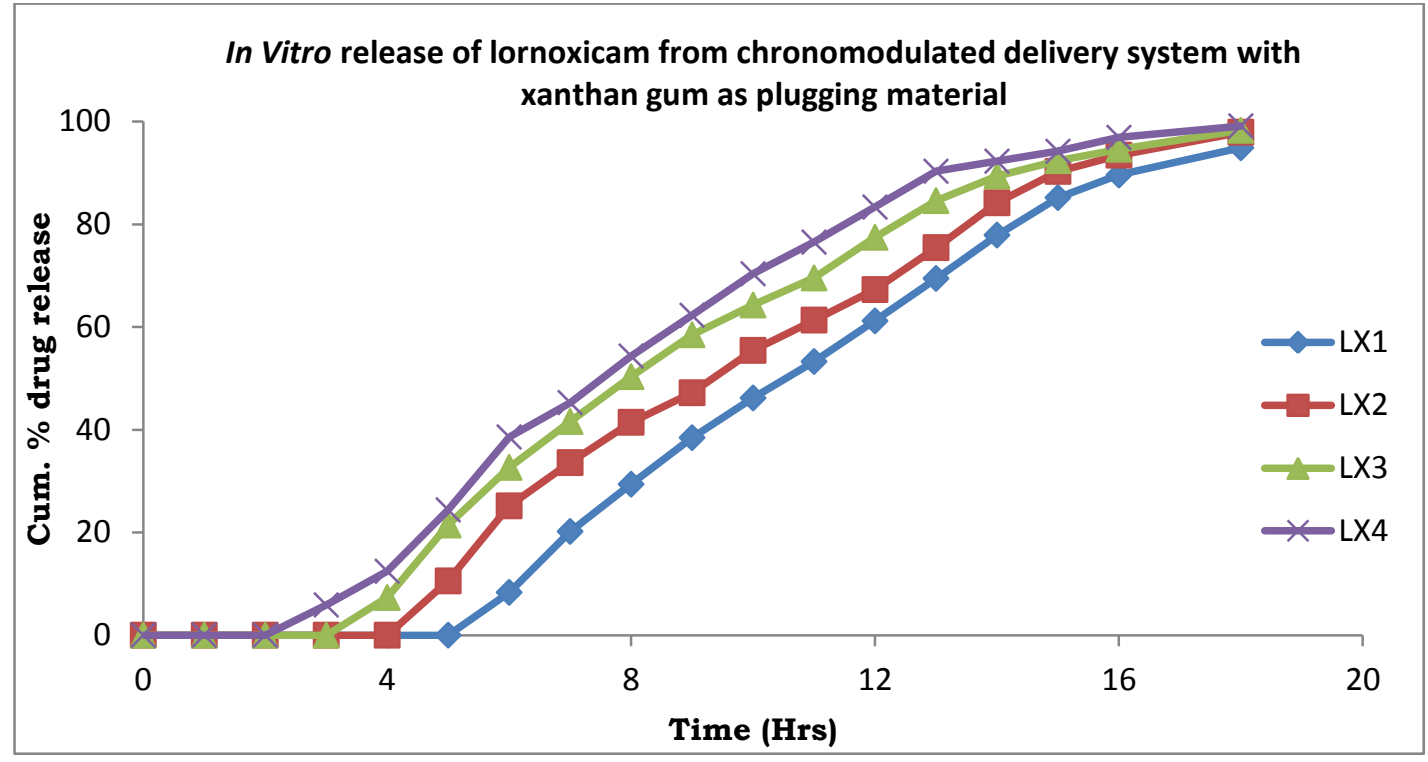

\section{CONCLUSION:}

The TPP cross-linked chitosan microspheres were found to be discrete, nearly spherical with rough surface characteristics. Particle size, encapsulation efficiency and release rate are dependent on the fabrication conditions of the microspheres. The developed microspheres were successfully utilized in the development of the chronomodulated delivery systems of lornoxicam. FT-IR, DSC and XRD studies revealed the compatibility of the drug with the excipients used. The exposure of hard gelatine capsule bodies with formaldehyde for a period of $12 \mathrm{hrs}$ was found to be optimum in maintaining the intactness of the capsule bodies in the simulated GI fluids. The ejection of the plug from the chrnomodulated delivery system depended on the nature of polymer and their concentration. The ejection of the plug delayed with increase in the polymer concentration and among the two polymers studied as plugging materials, guar gum exhibited better control in maintaining the lag time compared to xanthan gum. Overall, from the study it can be concluded that, the lag time can be efficiently tailored for the chronomodulated delivery of lornoxicam by varying the concentrations of the polymers in the hydrogel plug.

\section{ACKNOWLEDGEMENTS:}

The authors are thankful to Sun Pharma, Vadodara and Primex, Iceland for proving pure drug lornoxicam and chitosan respectively. The authors are also thankful to management of Navodaya Education Trust, Raichur, Karnataka for providing the facilities to carry out the research work.

Conflict of interest: The authors hereby declare that, there are no personnel or financial conflicts in the present research work. 


\section{REFERENCES:}

1. Bi-Botti CY. Chronopharmaceutics: Gimmick or clinically relevant approach to drug delivery - a review. J Control Rel 2004; 98(3):337-353.

2. Vyas SP, Sood A, P Venugopalan. Circadian rhythm and drug delivery design. Pharmazie 1997; 52: 815-20.

3. Youan BC. Chronopharmaceutics: Gimmick or clinically relevant approach to drug delivery?. J Control Rel 2004; 98:337-353

4. Cutolo M, Straub RH, Buttgereit F. Circadian rhythms of nocturnal hormones in rheumatoid arthritis: translation from bench to bedside. Ann Rheum Dis 2008; 67 (7):905-908

5. Medhi B , Patyar S, Wadha S, Prasad Byrav DS. Lornoxicam: a newer NSAID. IJPMR 2009; 20 (1): 27-31.

6. Merck \& Co. Inc. The Merck index. 13th ed. Whitehouse Station: Merck \& Co. Inc.; 2001.

7. Homdrum EM, Likar R, Nell G. Xefo® Rapid: a novel effective tool for pain treatment. Eur Surg 2006;38:342-52.

8. Kidd B, Frenzel W. A multicenter, randomized, double blind study comparing lornoxicam with diclofenac in osteoarthritis. J Rheumatol. 1996;23:1605-11.

9. Balfour JA, Fitton A, Barradell LB. Lornoxicam. A review of its pharmacology and therapeutic potential in the management of and inflammatory conditions. Drugs. 1996; 51:639-57.

10. Skjodt NM, Davies NM. Clinical pharmacokinetics of lornoxicam. A short half life oxicam. Clin Pharmacokinet. 1998;34:421-8.

11. Patil SS, Gupta VRM, Gupta KS, H Doddayya. Effect of pH, selected cyclodextrins and complexation methods on the solubility of lornoxicam. International Journal of Pharmacy and Pharmaceutical Sciences 2014; 6 (8): 324-27.

12. Anil K. Anal, Willem F. Stevens, Carmen Remu n'an-L'opez. Ionotropic cross-linked chitosan microspheres for controlled release of ampicillin. Int J Pharm 2006; 312: 166-73.

13. Asghar ALF, Chandran S. Multiparticulate formulation approach to colon specific drug delivery: current prosspectives. J Pharm Pharm Sci 2006;9:327-38

14. He P, Davis SS, Illum L. Chitosan microspheres prepared by spray drying. Int J Pharm 1999; 187: 53-65.

15. Remu n'an-L'opez C, Lorenzo-Lamosa ML, Vila-Jato JL, Alonso MJ. Development of new chitosan-cellulose Multicore microparticles for controlled drug delivery. Eur J Pharm Biopharm1998; 45: 49-56.

16. Buranachai T, Praphairaksit N, Muangsin N. Chitosan/Polyethylene Glycol Beads Crosslinked with Tripolyphosphate and Glutaraldehyde for Gastrointestinal Drug Delivery. AAPS PharmSciTech 2010; 11(3):1128-37.

17. Dhawan S, Singla AK, Sinha VR. Evaluation of Mucoadhesive Properties of Chitosan Microspheres Prepared by Different Methods. AAPS PharmSciTech 2004; 5 (4):1-7.

18. Garud A, Garud N. Preparation and Evaluation of Chitosan Microcapsules of Metronidazole using Tripolyphosphate Cross-linking Method. Dhaka Univ J Pharm Sci 2010; 9(2): 125-130

19. Reddy GS, B Sirisha, Uma maheshwar rao V, Vijaya lakshmi $\mathrm{P}$, Ajitha A. Formulation and in vitro evaluation of colon specific drug delivery of naproxen sodium by using pulsincap technology 2014; 5(1): 1751-60

20. Zhang LJ, Wu ZM, Zhou L, Guo XZ, Wei J, Ling L et al. HP55-coated capsule containing PLGA/RS nanoparticles for oral delivery of insulin. Intl J Pharm 2012; 425:1-8

21. Kaushik D, Sardana S, Mishra D. In-vitro Characterization and Cytotoxicity Analysis of 5- Fluorouracil loaded Chitosan Microspheres for Targeting Colon Cancer. Indian J Pharm Educ Res 2010; 44(3):274-82.

22. Ko JA, Park HJ, Hwang SJ, Park JB, Lee JS. Preparation and characterization of chitosan microparticles intended for controlled drug delivery. Int J Pharm 2002; 249: 165-174

23. Shu XZ, Zhu KJ. Controlled drug release properties of ionically cross-linked chitosan beads: the influence of anion structure. Int J Pharm 2002; 233: 217-225.

24. Harry G. Brittain. Profiles of Drug Substances, Excipients, and Related Methodology.In: Ahmaed MO, Al-Badr AA.Lornoxicam. 1st Ed, Volume 36; New York: Elsevier Publishers; 2011.p:206-39.

25. Mastiholimath VS, Dandgi PM, Jain SS, Gadad AP, Kulkarni AR. Time and $\mathrm{pH}$ dependent colon specific, pulsatile delivery of theophylline for the treatment of nocturnal asthma. Int $\mathrm{J}$ Pharm 2007; 328: 49-56. 